\title{
Theta operator for Hermitian modular forms over the Eisenstein field
}

\author{
Shoyu Nagaoka and Sho Takemori
}

\begin{abstract}
In this paper, we have investigated the $\bmod p$ kernel of the theta operator for Hermitian modular forms when the base field is the Eisenstein field.
\end{abstract}

\section{Introduction}

The first attempt to generalize Ramanujan's theta operator to a higher degree case was made in [1]. Subsequently, this generalization was developed by several researchers (e.g., [7], 2], [8]).

In the case of Siegel modular forms, the theta operator $\Theta$ is defined by $F=$ $\sum a_{F}(T) q^{T} \longrightarrow \Theta(F):=\sum \operatorname{det}(T) \cdot a_{F}(T) q^{T}$ for the generalized $q$-expansion $F=\sum a_{F}(T) q^{T}$. If we use the theta operator, some congruence property that satisfies Igusa's Siegel cusp form $\chi_{35}$ can be expressed as

$$
\left.\Theta\left(\chi_{35}\right) \equiv 0 \quad(\bmod 23) \quad(\text { cf. } 7]\right) .
$$

For a modular form $F$, the image $\Theta(F)$ is not necessarily a modular form in general. However, we know that, for a fixed prime number $p, \Theta(F)$ is congruent to a true modular form $G \bmod p$ under some condition on $p$ (cf. [1]) as follows:

$$
\Theta(F) \equiv G \quad(\bmod p)
$$

The modular form $G$ occasionally becomes zero, that is, $\Theta(F) \equiv 0(\bmod p)$. In this case, we say that $F$ is an element of the mod $p$ kernel of the theta operator. In the case of degree 2 Siegel modular forms, we have a number of examples of such forms. For example, Igusa's cusp form $\chi_{35}$ is an element of the mod 23 kernel of the theta operator as stated above. Moreover, the Siegel Eisenstein series $E_{12}^{(2)}$ and the Siegel theta series $\vartheta_{\mathcal{L}}^{(2)}$ associated with the Leech lattice $\mathcal{L}$ satisfy

$$
\Theta\left(E_{12}^{(2)}\right) \equiv \Theta\left(\vartheta_{\mathcal{L}}^{(2)}\right) \equiv 0 \quad(\bmod 23), \quad \text { ( e.g. cf. [13]) }
$$

In this paper, we shall show that similar phenomena exist in the case of Hermitian modular forms of degree 2 over the Eisenstein field $\mathbb{Q}(\sqrt{3} i)$. 
In 4], Dern and Krieg determined the structure of the graded rings of degree 2 Hermitian modular forms in the cases $\mathbb{Q}(i)$ (the Gaussian field) and $\mathbb{Q}(\sqrt{3} i)$ (the Eisenstein field). In the case of $\mathbb{Q}(\sqrt{3} i)$, they showed that there are two odd weight forms $\phi_{9}$ and $\phi_{45}$ in the set of generators. In this study, we shall show that

$$
(* *) \quad \Theta\left(\phi_{9}\right) \equiv 0 \quad(\bmod 2), \quad \Theta\left(\phi_{45}\right) \equiv 0 \quad(\bmod 11),
$$

in $\S 4.2$,

The congruence relations $(*)$ and $(* *)$ lead us to the following conjecture:

Any odd weight modular form with Borcherds product will be in the mod $p$ kernel of the theta operator for a suitable prime number $p$.

(Igusa's cusp form $\chi_{35}$ is a typical example of Borcherds product (cf. [5], Theorem 1.5.). Moreover, the modular forms $\phi_{9}$ and $\phi_{45}$ are constructed as Borcherds products (4], Corollary 3).)

The isometry classes of rank 12 Eisenstein lattices were classfied by Hentschel, Krieg and Nebe in [6]. They showed that there are exactly five isometry classes. According to their notation, we write the corresponding representative Gram matrices as $H_{i}(i=1, \ldots, 5)$. We show that the weight 12 Hermitian Eisenstein series $E_{12, \mathbb{Q}(\sqrt{3} i)}^{(2)}$ and the Hermitian theta series $\vartheta_{H_{i}}^{(2)}(i=4,5)$ satisfy

$$
\Theta\left(E_{12, \mathbb{Q}(\sqrt{3} i)}^{(2)}\right) \equiv \Theta\left(\vartheta_{H_{4}}^{(2)}\right) \equiv \Theta\left(\vartheta_{H_{5}}^{(2)}\right) \equiv 0 \quad(\bmod 11),
$$

where $H_{5}$ corresponds to the Hermitian Leech lattice (cf. $\S 3.3$ ) .

The congruence relation ( $\dagger \dagger$ ) and the corresponding result in the case of the Gaussian field (Theorem 8 in [8]) leads to the second conjecture:

Any Hermitian theta series associated with the Hermitian Leech lattice will be in the mod $p$ kernel of the theta operator for a suitable prime number $p$.

\section{Preliminaries}

\subsection{Notation}

The Hermitian upper half-space of degree 2 is given as

$$
\mathbb{H}_{2}:=\left\{Z \in M_{2}(\mathbb{C}) \mid \frac{1}{2 i}\left(Z-{ }^{t} \bar{Z}\right)>0\right\},
$$

where ${ }^{t} \bar{Z}$ denote the transpose, and the complex conjugate of $Z$. Let $\boldsymbol{K}$ be an imaginary quadratic number field with the discriminant $d_{\boldsymbol{K}}$ and the ring of integers

$$
\mathcal{O}_{\boldsymbol{K}}=\mathbb{Z}+\mathbb{Z} \omega, \quad \omega=\left\{\begin{array}{lll}
i \sqrt{\left|d_{\boldsymbol{K}}\right|} / 2, & \text { if } d_{\boldsymbol{K}} \equiv 0 \quad(\bmod 4) \\
\left(1+i \sqrt{\left|d_{\boldsymbol{K}}\right|}\right) / 2 & \text { if } d_{\boldsymbol{K}} \not \equiv 0 & (\bmod 4) .
\end{array}\right.
$$


Then

$$
\Gamma_{2}\left(\mathcal{O}_{K}\right):=\left\{M \in M_{4}\left(\mathcal{O}_{K}\right) \mid M J^{t} \bar{M}=J\right\}, \quad J:=\left(\begin{array}{cc}
0 & -E_{2} \\
E_{2} & 0
\end{array}\right),
$$

is called the Hermitian modular group of degree 2 over $\boldsymbol{K}$. The group $\Gamma_{2}\left(\mathcal{O}_{\boldsymbol{K}}\right)$ acts on $\mathbb{H}_{2}$ by the fractional linear transformation $Z \longmapsto M\langle Z\rangle:=(A Z+$ $B)(C Z+D)^{-1}, M=\left(\begin{array}{ll}A & B \\ C & D\end{array}\right)$. If $\Gamma \subset \Gamma_{2}\left(\mathcal{O}_{K}\right)$ is a subgroup of finite index and $\nu$ is an abelian character of $\Gamma$, the space $M_{k}(\Gamma, \nu), k \in \mathbb{Z}$ of the Hermitian modular forms of weight $k$ and character $\nu$ with respect to $\Gamma$ consists of all the holomorphic functions $F: \mathbb{H}_{2} \longrightarrow \mathbb{C}$ that satisfy

$$
\left.F\right|_{k} M(Z):=\operatorname{det}(C Z+D)^{-k} F(M\langle Z\rangle)=\nu(M) \cdot F(Z)
$$

for all $M=\left(\begin{array}{c}A B \\ C D\end{array}\right) \in \Gamma$. The subspace $S_{k}(\Gamma, \nu)$ of the cusp forms is characterized by the condition

$$
\left.F\right|_{k}\left(\begin{array}{cc}
{ }^{t} \bar{U} & 0 \\
0 & U^{-1}
\end{array}\right) \mid \Phi \equiv 0 \text { for all } U \in G L_{2}(\boldsymbol{K}),
$$

where $\Phi$ is the Siegel $\Phi$-operator. There is an exceptional automorphism of the Hermitian upper half-space

$$
I_{t r}: \mathbb{H}_{2} \longrightarrow \mathbb{H}_{2}, \quad Z \longmapsto{ }^{t} Z,
$$

satisfying

$$
M \circ I_{t r}=I_{t r} \circ \bar{M} \text { for all } \quad M \in \Gamma_{2}\left(\mathcal{O}_{K}\right) .
$$

The superscript sym (resp. skew) denotes the subspace of the symmetric (resp. skew-symmetric) Hermitian modular forms characterized by

$$
F \circ I_{t r}=F \quad \text { resp. } \quad F \circ I_{t r}=-F .
$$

Each $F \in M_{k}\left(\Gamma_{2}\left(\mathcal{O}_{K}\right), \operatorname{det}^{l}\right)$ possesses a Fourier expansion of the form

$$
F(Z)=\sum_{0 \leq H \in \Lambda_{2}\left(\mathcal{O}_{K}\right)} a(F ; H) \exp (2 \pi i \operatorname{tr}(H Z)),
$$

where

$$
\Lambda_{2}\left(\mathcal{O}_{\boldsymbol{K}}\right):=\left\{H=\left(\begin{array}{cc}
m & t \\
\bar{t} & n
\end{array}\right) \mid m, n \in \mathbb{Z}_{\geq 0}, t \in \frac{1}{\sqrt{d_{K}}} \mathcal{O}_{\boldsymbol{K}}=\mathcal{O}_{\boldsymbol{K}}^{\sharp}\right\} .
$$

For simplicity, we write the above Fourier expansion by $F=\sum a(F ; H) q^{H}$. We may regard that the right-hand side is an element of some formal power series ring $\mathbb{C} \llbracket q \rrbracket$ (cf. 11 , p.248).

Let $R$ be a subring of $\mathbb{C}$. We denote by $M_{k}\left(\Gamma_{2}\left(\mathcal{O}_{K}\right), \operatorname{det}^{l}\right)_{R}$ the $R$-module consisting of $F \in M_{k}\left(\Gamma_{2}\left(\mathcal{O}_{K}\right), \operatorname{det}^{l}\right)$ all of whose Fourier coefficients $a(F ; H)$ are in $R$. In this case, we may regard it as an element of $R \llbracket q \rrbracket$.

For a prime number $p$, we denote by $\mathbb{Z}_{(p)}$ the local ring at $p$ (i.e. the ring of $p$ integral rational numbers). For two elements $F_{i}=\sum a\left(F_{i} ; H\right) q^{H} \in \mathbb{Z}_{(p)} \llbracket q \rrbracket(i=$ $1,2)$, we write

$$
F_{1} \equiv F_{2} \quad(\bmod p)
$$

when $a\left(F_{1} ; H\right) \equiv a\left(F_{2} ; H\right)(\bmod p)$ holds for all $H \in \Lambda_{2}\left(\mathcal{O}_{K}\right)$. 


\subsection{Eisenstein series and theta series}

Examples of the Hermitian modular forms are given by the Hermitian Eisenstein series and the Hermitian theta series.

The Hermitian Eisenstein series of weight $k$ and degree 2 over $\boldsymbol{K}$ is defined by

$$
E_{k, \boldsymbol{K}}^{(2)}(Z):=\sum_{M=\left(\begin{array}{c}
* * \\
C
\end{array}\right):\left\{\left(\begin{array}{c}
* * * \\
0 *
\end{array}\right)\right\} \backslash \Gamma_{2}\left(\mathcal{O}_{K}\right)}(\operatorname{det}(M))^{k / 2} \operatorname{det}(C Z+D)^{-k},
$$

where $k>4$ is an even integer. It is known that $E_{k, \boldsymbol{K}}^{(2)} \in M_{k}\left(\Gamma_{2}\left(\mathcal{O}_{\boldsymbol{K}}\right) \text {, } \operatorname{det}^{-k / 2}\right)_{\mathbb{Q}}^{s y m}$ (cf. [4]). Moreover $E_{4, \boldsymbol{K}}^{(2)} \in M_{4}\left(\Gamma_{2}\left(\mathcal{O}_{\boldsymbol{K}}\right) \text {, } \operatorname{det}^{-2}\right)_{\mathbb{Q}}^{\text {sym }}$ is defined as the Maaß lift (cf. 4]). An explicit formula for $a\left(E_{k, \boldsymbol{K}}^{(2)} ; H\right)$ was given by Krieg [10] in the case when the class number of $\boldsymbol{K}$ was one.

The second example is the theta series. Let $S \in \operatorname{Her}_{m}(\boldsymbol{K})$ be a positive definite even matrix with respect to $\mathcal{O}_{K}$. The degree 2 Hermitian theta series associated with $S$ is defined by

$$
\vartheta^{(2)}(Z ; S):=\sum_{G \in M_{m, 2}\left(\mathcal{O}_{K}\right)} \exp \left(\pi i \operatorname{tr}\left(Z^{t} \bar{G} S G\right)\right) .
$$

If $S$ satisfies an additional condition $\operatorname{det}(S)=\left(2 / \sqrt{d_{\boldsymbol{K}}}\right)^{m}$ (i.e. $S$ is unimodular), then $m \equiv 0(\bmod 4)$ and

$$
\vartheta^{(2)}(Z ; S) \in M_{m}\left(S L_{4}(\boldsymbol{K}) \cap \Gamma_{2}\left(\mathcal{O}_{\boldsymbol{K}}\right), 1\right)_{\mathbb{Z}}^{\text {sym }},
$$

(cf. [3]).

\section{Hermitian modular forms over the Eisenstein field}

In the rest of this paper, we treat the case in which

$$
\boldsymbol{K}=\mathbb{Q}(\sqrt{3} i) \quad(\text { Eisenstein field }) .
$$

In this case

$$
d_{K}=-3, \quad \mathcal{O}_{K}=\mathbb{Z}+\omega \mathbb{Z}, \quad \omega=(1+\sqrt{3} i) / 2 .
$$

It is known that

$$
M_{k}\left(\Gamma_{2}\left(\mathcal{O}_{\boldsymbol{K}}\right), \operatorname{det}^{l}\right)= \begin{cases}M_{k}\left(S L_{4}(\boldsymbol{K}) \cap \Gamma_{2}\left(\mathcal{O}_{\boldsymbol{K}}\right), 1\right) & \text { if } k \equiv l \quad(\bmod 3), \\ \{0\} & \text { otherwise }\end{cases}
$$

In this case, the formal power series $\mathbb{C} \llbracket q \rrbracket$ introduced in $\S 2.1$ is explicitly given as follows:

$$
\mathbb{C} \llbracket q \rrbracket:=\mathbb{C}\left[\dot{q}_{12}^{ \pm 1}, \ddot{q}_{12}^{ \pm 1}\right] \llbracket \dot{q}_{11}, \dot{q}_{22} \rrbracket,
$$

where $\dot{q}_{12}:=\exp \left(2 \pi i\left(z_{12}-z_{21}\right) / 2 \sqrt{3} i\right), \ddot{q}_{12}:=\exp \left(2 \pi i\left(z_{12}+z_{21}\right) / 2\right), \dot{q}_{11}:=$ $\exp \left(2 \pi i z_{11}\right)$, and $\dot{q}_{22}:=\exp \left(2 \pi i z_{22}\right)$ for $Z=\left(\begin{array}{ll}z_{11} & z_{12} \\ z_{21} & z_{22}\end{array}\right)$. 


\subsection{Structure of the graded ring}

In the case $\boldsymbol{K}=\mathbb{Q}(\sqrt{3} i)$, Dern-Krieg $[4$ determined the structure of the graded ring

$$
\bigoplus_{k \in \mathbb{Z}} M_{k}\left(\Gamma_{2}\left(\mathcal{O}_{\boldsymbol{K}}\right), \operatorname{det}^{k}\right)=\bigoplus_{k \in \mathbb{Z}} M_{k}\left(S L_{4}(\boldsymbol{K}) \cap \Gamma_{2}\left(\mathcal{O}_{\boldsymbol{K}}\right), 1\right) \quad(\text { cf. (3.1) }) .
$$

Proposition 3.1. (迎, Corollary 3) Let $\boldsymbol{K}=\mathbb{Q}(\sqrt{3} i)$. Then there exist the Borcherds products

$$
\phi_{9} \in S_{9}\left(\Gamma_{2}\left(\mathcal{O}_{\boldsymbol{K}}\right), 1\right)^{\text {skew }} \quad \text { and } \quad \phi_{45} \in S_{45}\left(\Gamma_{2}\left(\mathcal{O}_{\boldsymbol{K}}\right), 1\right)^{\text {sym }} .
$$

We will study the mod $p$ properties of $\phi_{9}$ and $\phi_{45}$ in $\S 4.2$.

Theorem 3.2. (4 4 , Theorem 6$)$ Let $\boldsymbol{K}=\mathbb{Q}(\sqrt{3} i)$.

(1) The graded ring

$$
\bigoplus_{k \in \mathbb{Z}} M_{k}\left(\Gamma_{2}\left(\mathcal{O}_{\boldsymbol{K}}\right), \operatorname{det}^{k}\right)=\bigoplus_{k \in \mathbb{Z}} M_{k}\left(S L_{4}(\boldsymbol{K}) \cap \Gamma_{2}\left(\mathcal{O}_{\boldsymbol{K}}\right), 1\right)
$$

is generated by

$$
E_{4, \boldsymbol{K}}^{(2)}, \quad E_{6, \boldsymbol{K}}^{(2)}, \quad \phi_{9}, \quad E_{10, \boldsymbol{K}}^{(2)}, E_{12, \boldsymbol{K}}^{(2)}, \text { and } \phi_{45},
$$

where $\phi_{9}$ and $\phi_{45}$ are cusp forms given in Proposition 3.1 .

(2) The ideal of cusp forms in $\oplus M_{k}\left(\Gamma_{2}\left(\mathcal{O}_{\boldsymbol{K}}\right)\right.$, $\left.\operatorname{det}^{k}\right)$ is generated by

$$
\phi_{9}, f_{10}, f_{12}, \text { and } \phi_{45}
$$

where $f_{k}$ is defined by

$$
\begin{aligned}
& f_{10}:=E_{10, \boldsymbol{K}}^{(2)}-E_{4, \boldsymbol{K}}^{(2)} \cdot E_{6, \boldsymbol{K}}^{(2)} \in S_{10}\left(\Gamma_{2}\left(\mathcal{O}_{\boldsymbol{K}}\right), \mathrm{det}^{-5}\right)^{s y m} \\
& f_{12}:=E_{12, \boldsymbol{K}}^{(2)}-\frac{441}{691}\left(E_{4, \boldsymbol{K}}^{(2)}\right)^{3}-\frac{250}{691}\left(E_{6, \boldsymbol{K}}^{(2)}\right)^{2} \in S_{12}\left(\Gamma_{2}\left(\mathcal{O}_{\boldsymbol{K}}\right), 1\right)^{s y m}
\end{aligned}
$$

\subsection{Theta operator on Hermitian modular forms}

The theory of a theta operator on the Hermitian modular forms was developed by several researchers (e.g., [12, [8]).

We recall that the Fourier expansion of the Hermitian modular form can be regarded as an element of the formal power series ring $\mathbb{C} \llbracket q \rrbracket$ (cf. (3.2)). The theta operator over $\mathbb{C} \llbracket q \rrbracket$ is defined by

$$
\Theta: F=\sum a(F ; H) q^{H} \longmapsto \Theta(F):=\sum \operatorname{det}(H) \cdot a(F ; H) q^{H} .
$$

As stated in the introduction, $\Theta(F)$ is not necessarily a Hermitian modular form even if $F$ is. However we have the following result. 
Theorem 3.3. Let $\boldsymbol{K}=\mathbb{Q}(\sqrt{3} i)$ and $p$ be a prime number such that $p \geq 5$. For any $F \in M_{k}\left(\Gamma_{2}\left(\mathcal{O}_{\boldsymbol{K}}\right), \operatorname{det}^{k}\right)_{\mathbb{Z}_{(p)}}$, there is a cusp form

$$
G \in S_{k+p+1}\left(\Gamma_{2}\left(\mathcal{O}_{K}\right), \operatorname{det}^{k+p+1}\right)_{\mathbb{Z}_{(p)}}
$$

such that

$$
\Theta(F) \equiv G \quad(\bmod p) .
$$

Proof. The same type of statement in the case $\boldsymbol{K}=\mathbb{Q}(i)$ was given in [8], Theorem 3. A similar method using the Rankin-Cohen bracket is applicable for the case $\boldsymbol{K}=\mathbb{Q}(\sqrt{3} i)$ (e.g., [12]).

Example 3.4. Here we give an example of (3.3) in the case that $F=E_{4, \boldsymbol{K}}^{(2)}$ and $p=7$.

Let $f_{12}$ be the cusp form introduced in Theorem 3.2 (2). We normalize $f_{12}$ as

$$
\tilde{f}_{12}:=-\frac{691 \cdot 1847}{2^{13} \cdot 3^{6} \cdot 5^{3} \cdot 7^{2}} f_{12}, \quad a\left(\tilde{f}_{12},\left(\begin{array}{cc}
1 & 1 / \sqrt{3} i \\
-1 / \sqrt{3} i & 1
\end{array}\right)\right)=1 .
$$

Then all of the Fourier coefficients of $\tilde{f}_{12}$ are rational integers and

$$
\Theta\left(E_{4, \boldsymbol{K}}^{(2)}\right) \equiv \tilde{f}_{12} \quad(\bmod 7) .
$$

For example

$$
\left\{\begin{array}{c}
a\left(\Theta\left(E_{4, \boldsymbol{K}}^{(2)}\right) ;\left(\begin{array}{ll}
1 & x \\
\bar{x} & 1
\end{array}\right)\right)=4320 \\
a\left(\tilde{f}_{12} ;\left(\begin{array}{cc}
1 & x \\
\bar{x} & 1
\end{array}\right)\right)=1
\end{array}\right.
$$

for $x \in \boldsymbol{K}$ with $N(x)=1 / 3$, and

$$
\left\{\begin{array}{c}
a\left(\Theta\left(E_{4, \boldsymbol{K}}^{(2)}\right) ;\left(\begin{array}{ll}
1 & 0 \\
0 & 1
\end{array}\right)\right)=17280, \\
a\left(\tilde{f}_{12} ;\left(\begin{array}{ll}
1 & 0 \\
0 & 1
\end{array}\right)\right)=18
\end{array}\right.
$$

In the congruence $\Theta(F) \equiv G(\bmod p)$, the modular form $G$ sometimes vanishes identically, that is, $\Theta(F) \equiv 0(\bmod p)$. In this case, $F$ is called an element of the mod $p$ kernel of the theta operator.

The main purpose of this paper is to construct such modular forms.

\subsection{Hermitian theta series for Eisenstein lattices}

We still assume that $\boldsymbol{K}=\mathbb{Q}(\sqrt{3} i)$. We recall the definition of Eisenstein lattice. The lattice $\Lambda \subset \boldsymbol{K}^{r}$ is called an Eisenstein lattice of rank $r$ if there exist linearly independent vectors $b_{1}, \ldots, b_{r} \in \boldsymbol{K}^{r}$ such that 
(i) $\Lambda=\mathcal{O}_{\boldsymbol{K}} b_{1}+\cdots+\mathcal{O}_{\boldsymbol{K}} b_{r}$

(ii) $\operatorname{det}\left(\left\langle b_{j}, b_{k}\right\rangle\right)=(2 / \sqrt{3} i)^{r}$,

(iii) $\langle\lambda, \lambda\rangle \in 2 \mathbb{Z}$ for all $\lambda \in \Lambda$,

where $\langle\rangle:, \boldsymbol{K}^{r} \times \boldsymbol{K}^{r} \longrightarrow \boldsymbol{K}$ is the standard Hermitian scalar product defined by $\langle x, y\rangle={ }^{t} \bar{x} y$.

It is known that Eisenstein lattices exist only if $r$ is multiple of 4 (cf. $\S$ 2.2). Moreover, we have

$$
\vartheta^{(2)}(Z, S) \in M_{r}\left(S L_{4}(\boldsymbol{K}) \cap \Gamma_{2}\left(\mathcal{O}_{\boldsymbol{K}}\right), 1\right)_{\mathbb{Z}}^{\text {sym }}=M_{r}\left(\Gamma_{2}\left(\mathcal{O}_{\boldsymbol{K}}\right), \operatorname{det}^{-r / 2}\right)_{\mathbb{Z}}^{\text {sym }},
$$

where $S$ is the Gram matrix of an Eisenstein lattice (cf. [6], Proposition 2).

Hentschel-Krieg-Nebe classified isometry classes of Eisenstein lattices of rank 12 .

Theorem 3.5. ([6], Theorem 2). There are exactly five isometry classes of Eisenstein lattices rank 12 whose root lattices are $3 E_{8}, 4 E_{6}, 6 D_{4}, 12 A_{12}$, $\emptyset$ (i.e., the Hermitian Leech lattice).

According to [6], we write the corresponding Gram matrices as $H_{1}, \ldots, H_{5}$ and consider the theta series $\vartheta^{(2)}\left(Z, H_{i}\right) \in M_{12}\left(\Gamma_{2}\left(\mathcal{O}_{\boldsymbol{K}}\right), 1\right)_{\mathbb{Z}}^{\text {sym }}$.

The following identity is a special case of the analytic version of Siegel's main theorem:

\section{Proposition 3.6.}

$$
\begin{aligned}
E_{12, \boldsymbol{K}}^{(2)}= & -\frac{3 \cdot 7 \cdot 11 \cdot 13}{691 \cdot 809 \cdot 1847} \vartheta^{(2)}\left(Z, H_{1}\right)+\frac{2^{6} \cdot 5^{3} \cdot 7 \cdot 11 \cdot 13}{691 \cdot 809 \cdot 1847} \vartheta^{(2)}\left(Z, H_{2}\right) \\
& +\frac{2 \cdot 3^{8} \cdot 5^{2} \cdot 7 \cdot 11 \cdot 13}{691 \cdot 809 \cdot 1847} \vartheta^{(2)}\left(Z, H_{3}\right)+\frac{2^{15} \cdot 3^{2} \cdot 5^{2} \cdot 7 \cdot 13}{691 \cdot 809 \cdot 1847} \vartheta^{(2)}\left(Z, H_{4}\right) \\
& +\frac{2^{8} \cdot 3^{9} \cdot 5}{691 \cdot 809 \cdot 1847} \vartheta^{(2)}\left(Z, H_{5}\right) .
\end{aligned}
$$

Proof. The identity is obtained by the direct calculation of the Fourier coefficients of $E_{12, \boldsymbol{K}}^{(2)}$ and $\vartheta^{(2)}\left(Z, H_{i}\right)$.

\subsection{Sturm bound for Hermitian modular forms over the Eienstein field}

To prove congruences among Hermitian modular forms, we recall some results of the Sturm bound in the case of Hermitian modular forms over the Eisenstein field.

Theorem 3.7. ( $[8]$, Theorem 2). Let $\boldsymbol{K}=\mathbb{Q}(\sqrt{3} i)$ and $p$ be a prime number such that $p \geq 5$. If a Hermitian modular form $F \in M_{k}\left(\Gamma_{2}\left(\mathcal{O}_{K}\right) \text {, } \operatorname{det}^{k}\right)_{\mathbb{Z}_{(p)}}^{s y m}$, with even weight $k$ satisfies

$$
a(F ; H) \equiv 0 \quad(\bmod p)
$$

for all $H=\left(\begin{array}{c}m * \\ * n\end{array}\right) \in \Lambda_{2}\left(\mathcal{O}_{K}\right)$ with $m, n \leq\left[\frac{k}{9}\right]$, then

$$
F \equiv 0 \quad(\bmod p) \text {. }
$$




\section{Main results}

As stated in the introduction, the main purpose of this paper is to constuct some examples of Hermitian modular forms in the mod $p$ kernel of the theta operator.

\subsection{Hermitian theta series}

In $\S 2.2$, we considered the Hermitian theta series $\vartheta^{(2)}\left(Z, H_{i}\right)$ where $H_{i}(i=$ $1, \ldots, 5)$ are the Hermitian matrices corresponding to the representatives of Eisenstein lattices of rank 12. The first main result shows that two of $\vartheta^{(2)}\left(Z, H_{i}\right)$ are in the mod 11 kernel of the theta operator.

Theorem 4.1. We have

$$
\Theta\left(E_{12, \boldsymbol{K}}^{(2)}\right) \equiv \Theta\left(\vartheta^{(2)}\left(Z, H_{4}\right)\right) \equiv \Theta\left(\vartheta^{(2)}\left(Z, H_{5}\right)\right) \equiv 0 \quad(\bmod 11),
$$

in particular, $\vartheta^{(2)}\left(Z, H_{4}\right)$ and $\vartheta^{(2)}\left(Z, H_{5}\right)$ are in the mod 11 kernel of the theta operator.

Proof. The statement that $\Theta\left(E_{12, \boldsymbol{K}}^{(2)}\right) \equiv 0(\bmod 11)$ is a consequence of $[8]$, Theorem 5. Next we shall show that

$$
\Theta\left(\vartheta^{(2)}\left(Z, H_{5}\right)\right) \equiv 0 \quad(\bmod 11) .
$$

We apply the setting in Theorem 3.3 to

$$
\left\{\begin{array}{l}
F=\vartheta^{(2)}\left(Z, H_{5}\right) \in M_{12}\left(\Gamma_{2}\left(\mathcal{O}_{K}\right), \operatorname{det}^{-6}\right)_{\mathbb{Z}}=M_{12}\left(\Gamma_{2}\left(\mathcal{O}_{K}\right), \operatorname{det}^{12}\right)_{\mathbb{Z}} \\
p=11
\end{array}\right.
$$

Then there is a modular form $G \in S_{24}\left(\Gamma_{2}\left(\mathcal{O}_{K}\right) \text {, } \operatorname{det}^{24}\right)_{\mathbb{Z}_{(11)}}$ such that

$$
\Theta\left(\vartheta^{(2)}\left(Z, H_{5}\right)\right) \equiv G \quad(\bmod 11) .
$$

The numerical data (Table 1 in $\S\left[\right.$ 5.1) on the Fourier coefficients $a\left(\vartheta^{(2)}\left(Z, H_{5}\right) ; H\right)$ shows that

$$
\operatorname{det}(H) \cdot a(G ; H) \equiv 0 \quad(\bmod 11)
$$

for all $H=\left(\begin{array}{c}m * \\ * n\end{array}\right)$ with $n, m \leq\left[\frac{24}{9}\right]=2$. By Sturm's bound (Theorem 3.7), we obtain

$$
\Theta\left(\vartheta^{(2)}\left(Z, H_{5}\right)\right) \equiv G \equiv 0 \quad(\bmod 11)
$$

Finally, we prove $\Theta\left(\vartheta^{(2)}\left(Z, H_{4}\right)\right) \equiv 0(\bmod 11)$. We recall that $\Theta\left(E_{12, \boldsymbol{K}}^{(2}\right) \equiv 0$ $(\bmod 11)$. However, it follows from Proposition 3.6 that

$$
7 \vartheta^{(2)}\left(Z, H_{4}\right)+5 \vartheta^{(2)}\left(Z, H_{5}\right) \equiv E_{12, \boldsymbol{K}}^{(2)}(\bmod 11) .
$$

Since $\Theta\left(\vartheta^{(2)}\left(Z, H_{5}\right)\right) \equiv \Theta\left(E_{12, \boldsymbol{K}}^{(2)}\right) \equiv 0(\bmod 11)$, we obtain

$$
\Theta\left(\vartheta^{(2)}\left(Z, H_{4}\right)\right) \equiv 0 \quad(\bmod 11) .
$$


A congruence relation similar to the above Theorem holds if $\boldsymbol{K}$ is the Gaussian field (Theorem 8 in [8]). These facts lead us the following conjecture:

Any Hermitian theta series associated with the Hermitian Leech lattice will be in the mod $p$ kernel of the theta operator for a suitable prime number $p$.

\subsection{Odd weight forms}

In $\S$ 3.1, we saw a set of generators of the graded ring $\oplus M_{k}\left(\Gamma_{2}\left(\mathcal{O}_{\boldsymbol{K}}\right), \operatorname{det}^{k}\right)$ (cf. Theorem 3.2). There are two modular forms with odd weight in the set of generators. The second main result shows that these forms are in the kernel of the theta operator.

Let $\phi_{9}$ and $\phi_{45}$ be odd weight modular forms given in Theorem 3.3. We assume that they are normalized as

$$
a\left(\phi_{9} ;\left(\begin{array}{cc}
1 & -1 / \sqrt{3} i \\
1 / \sqrt{3} i & 1
\end{array}\right)\right)=a\left(\phi_{45} ;\left(\begin{array}{ll}
3 & 1 \\
1 & 4
\end{array}\right)\right)=1
$$

(i.e., the first Fourier coefficient is equal to one).

Now we use the following abbreviations for $H \in \Lambda_{2}\left(\mathcal{O}_{K}\right)$ :

$$
H=\left(\begin{array}{cc}
m & (a+b \sqrt{3} i) / 2 \sqrt{3} i \\
(-a+b \sqrt{3} i) / 2 \sqrt{3} i & n
\end{array}\right)=:(m, n, a, b)
$$

and

$$
q^{(m, n, a, b)}:=q^{H}=\exp (2 \pi i \operatorname{tr}(H Z)) .
$$

By numerical computation, a few Fourier coefficients of $\phi_{9}$ are given as follows:

$$
\begin{aligned}
\phi_{9}=q^{(1,1,-2,0)} & -q^{(1,1,-1,-1)}-q^{(1,1,-1,1)} \\
& +q^{(1,1,1,-1)}+q^{(1,1,1,1)}-q^{(1,1,2,0)}+\sum_{H} a\left(\phi_{9} ; H\right) q^{H} .
\end{aligned}
$$

In the last summation, $H$ runs over the elements of $\Lambda_{2}\left(\mathcal{O}_{K}\right)_{>0}$ such that $H=$ $\left(\begin{array}{c}m * \\ * n\end{array}\right)$ with $\max (m, n)>1$.

The coefficients of $\phi_{45}$ are given as follows:

$$
\begin{aligned}
\phi_{45}= & q^{(3,4,-3,-1)}-q^{(3,4,-3,1)}-q^{(3,4,0,2)} \\
& +q^{(3,4,3,-1)}-q^{(3,4,3,1)}-q^{(4,3,-3,-1)}+q^{(4,3,-3,1)} \\
& +q^{(4,3,0,-2)}-q^{(4,3,0,2)}-q^{(4,3,3,-1)}+q^{(4,3,3,1)} \\
& +\sum_{H} a\left(\phi_{45} ; H\right) q^{H},
\end{aligned}
$$

where $H$ runs over elements of $\Lambda_{2}\left(\mathcal{O}_{K}\right)_{>0}$ such that $H=\left(\begin{array}{c}m * \\ * n\end{array}\right)$ with $\max (m, n)>$ 4. (Further examples of $a\left(\phi_{9} ; H\right)$ and $a\left(\phi_{45} ; H\right)$ are given in $\S 5$.) 
Lemma 4.2. We have

$$
\phi_{9} \in S_{9}\left(\Gamma_{2}\left(\mathcal{O}_{K}\right), 1\right)_{\mathbb{Z}}^{\text {skew }} \quad \text { and } \quad \phi_{45} \in S_{45}\left(\Gamma_{2}\left(\mathcal{O}_{K}\right), 1\right)_{\mathbb{Z}}^{\text {sym }} .
$$

Moreover, let $\phi=\phi_{9}$ or $\phi_{45}$ and $f \in R \llbracket q \rrbracket$ for some ring $R \subset \mathbb{C}$, if there exists $g \in \mathbb{C} \llbracket q \rrbracket$ such that $f=\phi g$, then we have

$$
g \in R \llbracket q \rrbracket .
$$

Proof. The first statement of integrality follows from the fact that $\phi_{9}$ and $\phi_{45}$ are Borcherds products. The second statement follows from the above explicit Fourier expansions of $\phi_{9}$ and $\phi_{45}$ because they are monic if we define a suitable order of monomials. by

We denote by $\mathbb{S}_{2}$ the Siegel upper-half space of degree 2. This is characterized

$$
\mathbb{S}_{2}=\left\{Z \in \mathbb{H}_{2} \mid{ }^{t} Z=Z\right\} .
$$

Lemma 4.3. Let $p \geq 3$ be a prime number and $F \in M_{k}\left(\Gamma_{2}\left(\mathcal{O}_{\boldsymbol{K}}\right), \operatorname{det}^{k}\right)_{\mathbb{Z}_{(p)}}^{\text {sym }}$ with $k$ odd. If $\left.F\right|_{\mathbb{S}_{2}} \equiv 0(\bmod p)$, then there exists

$$
G \in M_{k-18}\left(\Gamma_{2}\left(\mathcal{O}_{K}\right), \operatorname{det}^{k-18}\right)_{\mathbb{Z}_{(p)}}^{\text {sym }}
$$

such that $F \equiv \phi_{9}^{2} G(\bmod p)$.

Proof. By Lemma 2 of [4] and Lemma 4.2, there exists

$$
h \in M_{k-45}\left(\Gamma_{2}\left(\mathcal{O}_{\boldsymbol{K}}\right), \operatorname{det}^{k-45}\right)_{\mathbb{Z}_{(p)}}^{\text {sym }}
$$

such that $F=\phi_{45} h$. It is known that $\left.\phi_{45}\right|_{\mathbb{S}_{2}}=\chi_{10} \chi_{35}$ where $\chi_{k}$ are Igusa's Siegel cusp forms of degree 2. Considering the Fourier expansion of $\chi_{10} \chi_{35}$, we see that $\left.h\right|_{\mathbb{S}_{2}} \equiv 0(\bmod p)$. Since there exists $J \in M_{k-45}\left(\Gamma_{2}\left(\mathcal{O}_{K}\right), \operatorname{det}^{k-45}\right)_{\mathbb{Z}_{(p)}}^{\text {sym }}$ such that $\left.p J\right|_{\mathbb{S}_{2}}=\left.h\right|_{\mathbb{S}_{2}}$, we have

$$
\left.\left(F-p \phi_{45} J\right)\right|_{\mathbb{S}_{2}}=0 .
$$

By [4] and Lemma 4.2, there exists

$$
G^{\prime} \in M_{k-9}\left(\Gamma_{2}\left(\mathcal{O}_{\boldsymbol{K}}\right), \operatorname{det}^{k-9}\right)_{\mathbb{Z}_{(p)}}^{\text {skew }}
$$

such that $F \equiv \phi_{9} G^{\prime}(\bmod p)$. Again by Lemma 2 of [4] and Lemma 4.2, there is $G \in M_{k-18}\left(\Gamma_{2}\left(\mathcal{O}_{K}\right) \text {, } \operatorname{det}^{k-18}\right)_{\mathbb{Z}_{(p)}}^{\text {sym }}$ such that $G^{\prime}=\phi_{9} G$.

Lemma 4.4. Let $F=\sum_{m, n \geq 0} a_{m, n}\left(F ; \dot{q}_{12}, \ddot{q}_{12}\right) q_{11}^{m} q_{22}^{n} \in \mathbb{Z}_{(p)} \llbracket q \rrbracket$ (cf. (3.2)). Suppose that there is $G \in \mathbb{Z}_{(p)} \llbracket q \rrbracket$ such that $F \equiv \phi_{9} G(\bmod p)$ and

$$
a_{m, n}\left(F ; \dot{q}_{12}, \ddot{q}_{12}\right) \equiv 0 \quad(\bmod p)
$$

for all $m, n \leq N$. Then we have

$$
a_{m, n}\left(G ; \dot{q}_{12}, \ddot{q}_{12}\right) \equiv 0 \quad(\bmod p)
$$

for all $m, n \leq N-1$. 
Proof. As an element of $\mathbb{Z}_{(p)} \llbracket q \rrbracket, \phi_{9}$ is given by

$$
\begin{aligned}
\phi_{9} & =\dot{q}_{11} \ddot{q}_{22}\left(\dot{q}_{12}^{-2}-\dot{q}_{12}^{-1} \ddot{q}_{12}^{-1}-\dot{q}_{12}^{-1} \ddot{q}_{12}+\dot{q}_{12} \ddot{q}_{12}^{-1}+\dot{q}_{12} \ddot{q}_{12}-\ddot{q}_{12}^{2}\right) \\
& \left.+\sum_{\max (m, n)>1} a\left(\phi_{9} ; \dot{q}_{12}, \ddot{q}_{12}\right) \dot{q}_{11}^{m} \dot{q}_{22}^{n}, \quad \text { (cf. (3.2) }\right) .
\end{aligned}
$$

The statement follows from this expression.

Proposition 4.5. Let $p \geq 3$ be a prime number. Suppose that $k \geq 0$ is odd and $F \in M_{k}\left(\Gamma_{2}\left(\mathcal{O}_{K}\right), \operatorname{det}^{k}\right)_{\mathbb{Z}_{(p)}}$. If

$$
a(F ; H) \equiv 0 \quad(\bmod p)
$$

for any $H=\left(\begin{array}{c}m * \\ * n\end{array}\right) \in \Lambda_{2}\left(\mathcal{O}_{K}\right)_{\geq 0}$ such that $m, n \leq[k / 9]-1$. Then we have $a(F ; H) \equiv 0(\bmod p)$ for any $H \in \Lambda\left(\mathcal{O}_{\boldsymbol{K}}\right)_{\geq 0}$, namely, $F \equiv 0(\bmod p)$.

Proof. Since the proof is similar, we prove the case only when $F$ is symmetric. We prove the statement by induction on $k$.

First, let $k=45$ and $F$ be a constant multiple of $\phi_{45}$. (Note that $\phi_{9}$ is skew symmetric.) Then the statement follows from the Fourier expansion of $\phi_{45}$. Next we assume that $k>45$ and the statement is true for smaller weights. By assumption and Sturm bound for the Siegel modular case (cf. 9]), we have $\left.F\right|_{\mathbb{S}_{2}} \equiv 0$ $(\bmod p)$. By Lemma 4.3. there exists $G \in M_{k-18}\left(\Gamma_{2}\left(\mathcal{O}_{\boldsymbol{K}}\right), \operatorname{det}^{k-18}\right)_{\mathbb{Z}_{(p)}}^{\text {sym }}$ such that $F \equiv \phi_{9}^{2} G(\bmod p)$. By Lemma 4.4 for any $H=\left(\begin{array}{c}m * \\ * n\end{array}\right) \in \Lambda\left(\mathcal{O}_{K}\right)_{\geq 0}$, we have $a(G ; H) \equiv 0(\bmod p)$ if $m, n \leq[k / 3]-3=[(k-18) / 9]-1$. By induction hypothesis, we have $G \equiv 0(\bmod p)$.

The second main result can be stated as follows:

Theorem 4.6. We have the following congruence relations:

(1) $\Theta\left(\phi_{9}\right) \equiv 0(\bmod 2)$

(2) $\Theta\left(\phi_{45}\right) \equiv 0(\bmod 11)$.

Proof. (1) We consider the Hermitian Rankin-Cohen bracket $\left[\phi_{9}, E_{4, \boldsymbol{K}}^{(2)}\right]$, where $\phi_{9}$ and $E_{4, \boldsymbol{K}}^{(2)}$ are forms in the generators of the graded ring $\oplus M_{k}\left(\Gamma_{2}\left(\mathcal{O}_{\boldsymbol{K}}\right)\right.$, $\left.\operatorname{det}^{k}\right)$. The Hermitian Rankin-Cohen bracket [,] defined similarly as in the case of Siegel modular forms (cf. [1], 12]). Using the fact that $E_{4, \boldsymbol{K}}^{(2)} \equiv 1\left(\bmod 2^{4}\right)$ and explicit expression of $\left[\phi_{9}, E_{4, \boldsymbol{K}}^{(2)}\right]$, we have the following expression:

$$
\left[\phi_{9}, E_{4, \boldsymbol{K}}^{(2)}\right]=2^{3} c_{0} \cdot \Theta\left(\phi_{9}\right) E_{4, \boldsymbol{K}}^{(2)}+2^{m} c_{1} \cdot P
$$

where $c_{i} \in \mathbb{Z}_{(2)} \quad\left(\left(c_{0}, 2\right)=1\right), 4 \leq m \in \mathbb{Z}$, and $P$ is a Fouirer series in $\mathbb{Z} \llbracket q \rrbracket$. We set $G_{1}:=\left(2^{3} c_{0}\right)^{-1}\left[\phi_{9}, E_{4, \boldsymbol{K}}^{(2)}\right]$. Then we obtain $G_{1} \in S_{15}\left(\Gamma_{2}\left(\mathcal{O}_{\boldsymbol{K}}\right), \operatorname{det}^{15}\right)_{\mathbb{Z}_{(2)}}$ and

$$
\Theta\left(\phi_{9}\right) \equiv G_{1} \quad(\bmod 2) .
$$


By Theorem 3.2, we can write

$$
G_{1}=\gamma \cdot E_{6, K}^{(2)} \phi_{9} \quad\left(\gamma \in \mathbb{Z}_{(2)}\right) .
$$

Computing the Fourier coefficients of $\Theta\left(\phi_{9}\right)$ and $G_{1}$ at $H=\left(\begin{array}{cc}1 & i / \sqrt{3} \\ -i / \sqrt{3} & 1\end{array}\right)$, we obtain

$$
\left\{\begin{array}{l}
a\left(\Theta\left(\phi_{9}\right) ; H\right)=\frac{2}{3} \equiv 0 \quad(\bmod 2) \\
a\left(G_{1} ; H\right)=\gamma
\end{array}\right.
$$

This implies $\gamma \equiv 0(\bmod 2)$, and we get

$$
\Theta\left(\phi_{9}\right) \equiv G_{1} \equiv 0 \quad(\bmod 2) .
$$

(2) By Theorem 3.3, there is a modular form $G_{2} \in S_{57}\left(\Gamma_{(} \mathcal{O}_{K}\right)$, det $\left.{ }^{57}\right)_{\mathbb{Z}_{(11)}}$ such that

$$
\Theta\left(\phi_{45}\right) \equiv G_{2} \quad(\bmod 11) .
$$

By Table 3 in $\S 5$, we see that

$$
a\left(G_{2} ; H\right) \equiv 0 \quad(\bmod 11)
$$

for all $H=\left(\begin{array}{c}m * \\ * n\end{array}\right) \in \Lambda_{2}\left(\mathcal{O}_{K}\right)_{\geq 0}$ with $m, n \leq[57 / 9]-1=5$. It follows from Proposition 4.5 that

$$
a\left(G_{2} ; H\right) \equiv 0 \quad(\bmod 11)
$$

for all $H \in \Lambda_{2}\left(\mathcal{O}_{K}\right)$. This implies

$$
\Theta\left(\phi_{45}\right) \equiv G_{2} \equiv 0 \quad(\bmod 11) .
$$

Finally, we refer to the mod $p$ property of Borcherds product. In the case of Siegel modular forms, we know that Igusa's cusp form $\chi_{35}$, which is a typical example of Borcherds product, represents an element in the mod 23 kernel of the theta operator. The above results in Theorem 4.6 lead us to the following conjecture.

Any modular form of odd weight coming from Borcherds product will be in the mod $p$ kernel of the theta operator for a suitable prime number $p$.

\section{Tables}

In this section, we summarize the tables of Fourier coefficients that are needed in the proof of statements in the previous sections.

As in $\S 4$, we use the following abbreviation:

$$
\Lambda_{2}\left(\mathcal{O}_{K}\right) \ni H=\left(\begin{array}{cc}
m & (a+b \sqrt{3} i) /(2 \sqrt{3} i) \\
(-a+b \sqrt{3} i) /(2 \sqrt{3} i) & n
\end{array}\right)=:(m, n, a, b) .
$$




\subsection{Fourier coefficients of Hermitian theta series}

In $\S 4.1$ we considered the degree 2 theta series $\vartheta^{(2)}\left(Z, H_{i}\right)$ for rank 12 Eisenstein lattices $H_{i}$. The fifth matrix $H_{5}$ corresponds to the Hermitian Leech lattice (cf. Proposition 3.5). The following table concerns the Fourier coefficients $a\left(\vartheta^{(2)}\left(Z, H_{5}\right) ; H\right)$.

Table 1: Fourier coefficient $a\left(\vartheta^{(2)}\left(Z, H_{5}\right) ; H\right)$

\begin{tabular}{lll}
\hline$H$ & $3 \operatorname{det}(H)$ & $a\left(\vartheta^{(2)}\left(Z, H_{5}\right) ; H\right)$ \\
\hline$(2,2,6,0)$ & 3 & 0 \\
\hline$(2,2,5,1)$ & 5 & 0 \\
\hline$(2,2,4,0)$ & 8 & $175134960=2^{4} \cdot 3^{7} \cdot 5 \cdot 7 \cdot 11 \cdot 13$ \\
\hline$(2,2,3,1)$ & 9 & $553512960=2^{12} \cdot 3^{3} \cdot 5 \cdot 7 \cdot 11 \cdot 13$ \\
\hline$(2,2,2,0)$ & 11 & $4075868160=2^{12} \cdot 3^{7} \cdot 5 \cdot 7 \cdot 13$ \\
\hline$(2,2,0,0)$ & 12 & $980755760=2^{7} \cdot 3^{7} \cdot 5 \cdot 7^{2} \cdot 11 \cdot 13$ \\
\hline
\end{tabular}

\subsection{Fourier coefficients of odd weight forms}

Let $\phi_{9}$ be the modular form given in Proposition 3.1 which is the first odd weight generator of the graded ring. We take a normalization

$$
a\left(\phi_{9} ;(1,1,-2,0)\right)=1
$$

as in $\S 4$.

For $n \in \mathbb{Z}_{\geq 1}$, we set

$$
K_{n}:=(1, n, 2,0) \in \Lambda_{2}\left(\mathcal{O}_{\boldsymbol{K}}\right) .
$$

Any non-zero Fourier coefficient $a\left(\phi_{9} ; H\right)$ coincides with $a\left(\phi_{9} ; K_{n}\right)$ for $K_{n}$ with $\operatorname{det}(H)=\operatorname{det}\left(K_{n}\right)$ up to sign. 
Table 2: Fourier coefficients $a\left(\phi_{9} ; K_{n}\right)$ for $n \leq 20$

\begin{tabular}{lll}
\hline$n$ & $3 \operatorname{det}\left(K_{n}\right)$ & $a\left(\phi_{9} ; K_{n}\right)$ \\
\hline 1 & 2 & -1 \\
\hline 2 & 5 & $16=2^{4}$ \\
\hline 3 & 8 & $-104=-2^{3} \cdot 13$ \\
\hline 4 & 11 & $320=-2^{6} \cdot 5$ \\
\hline 5 & 14 & $-260=-2^{2} \cdot 5 \cdot 13$ \\
\hline 6 & 17 & $-1248=-2^{5} \cdot 3 \cdot 13$ \\
\hline 7 & 20 & $3712=2^{7} \cdot 29$ \\
\hline 8 & 23 & $-1664=-2^{7} \cdot 13$ \\
\hline 9 & 26 & $-6890=-2 \cdot 5 \cdot 13 \cdot 53$ \\
\hline 10 & 29 & $7280=2^{4} \cdot 5 \cdot 7 \cdot 13$ \\
\hline 11 & 32 & $5568=2^{6} \cdot 3 \cdot 29$ \\
\hline 12 & 35 & $4160=2^{6} \cdot 5 \cdot 13$ \\
\hline 13 & 38 & $-33176=-2^{3} \cdot 11 \cdot 13 \cdot 29$ \\
\hline 14 & 41 & $-4640=-2^{5} \cdot 5 \cdot 29$ \\
\hline 15 & 44 & $74240=2^{9} \cdot 5 \cdot 29$ \\
\hline 16 & 47 & $-29824=-2^{7} \cdot 233$ \\
\hline 17 & 50 & $-14035=-5 \cdot 7 \cdot 401$ \\
\hline 18 & 53 & $-54288=-2^{4} \cdot 3^{2} \cdot 13 \cdot 29$ \\
\hline 19 & 56 & $-27040=-2^{5} \cdot 5 \cdot 13^{2}$ \\
\hline 20 & 59 & $142720=2^{7} \cdot 5 \cdot 223$ \\
\hline
\end{tabular}

Remark 5.1. From Table 2, we can see that the prime numbers 13 and 29 appear frequently as the prime factors of $a\left(\phi_{9} ; K_{n}\right)$. It has been confirmed that the phenomenon occurs for a wide range of $n$. 
The second odd weight form in the set of generators is $\phi_{45}$.

Table 3: Fourier coefficients $a\left(\phi_{45} ; H\right)$

\begin{tabular}{lll}
\hline$H$ & $3 \operatorname{det}(H)$ & $a\left(\phi_{45} ; H\right)$ \\
\hline$(3,4,0,2)$ & 33 & 1 \\
\hline$(3,5,0,-2)$ & 42 & $88=2^{3} \cdot 11$ \\
\hline$(3,6,0,2)$ & 51 & $3740=2^{2} \cdot 5 \cdot 11 \cdot 17$ \\
\hline$(4,5,-5,1)$ & 53 & $16038=2 \cdot 3^{6} \cdot 11$ \\
\hline$(4,5,0,-2)$ & 57 & $95931=3^{3} \cdot 11 \cdot 17 \cdot 19$ \\
\hline$(4,6,-5,1)$ & 65 & $681615=3^{6} \cdot 5 \cdot 11 \cdot 17$ \\
\hline$(4,6,0,2)$ & 69 & $720940=2^{2} \cdot 5 \cdot 11 \cdot 29 \cdot 113$ \\
\hline$(5,6,-7,-1)$ & 77 & $47271276=2^{2} \cdot 3^{6} \cdot 13 \cdot 29 \cdot 43$ \\
\hline$(5,6,-6,-2)$ & 78 & $13709344=2^{5} \cdot 11 \cdot 17 \cdot 29 \cdot 79$ \\
\hline$(5,6,-5,1)$ & 83 & $62772732=2^{2} \cdot 3^{6} \cdot 11 \cdot 19 \cdot 103$ \\
\hline$(5,6,-3,-1)$ & 87 & $835953624=2^{3} \cdot 3^{2} \cdot 11 \cdot 127 \cdot 8311$ \\
\hline
\end{tabular}

\section{References}

[1] S. Böcherer and S. Nagaoka, On mod $p$ properties of Siegel modular forms, Math. Ann., 338, 421-433(2007)

[2] S. Böcherer, H. Kodama and S. Nagaoka, On the kernel of the theta operator $\bmod p$, manuscripta math., 156, 149-169(2017)

[3] D.M. Cohen and H.L. Resnikoff, Hermitian quadratic forms and Hermitian modular forms, Pac. J. Math., 76, 329-337(1978)

[4] T. Dern and A. Krieg, Graded rings of Hermitian modular forms of degree 2, manuscripta math., 110, 251-272(2003)

[5] V.A. Gritsenko and V.V Nikulin, Igusa modular forms and simplest Lorentzian Kac-Moody algebras, Math. Sbornik, 187, 1601-1641(1996)

[6] M. Hentschel, A. Krieg, and G. Nebe, On the classification of lattices over $\mathbb{Q}(\sqrt{-3})$, which are even unimodular $\mathbb{Z}$-lattices, Abh. Math. Semin. Univ. Hambg., 80, 183-192(2010)

[7] T. Kikuta, H. Kodama and S. Nagaoka, Note on Igusas cusp form of weight 35, Rocky Mountain J. of Math. 45, 963-972(2015)

[8] T. Kikuta and S. Nagaoka, On the theta operator for Hermitian modular forms of degree 2, Abh. Math. Semin. Univ. Hambg, 87, 145-163(2017)

[9] T. Kikuta and S. Takemori, Sturm bounds for Siegel modular forms of degree 2 and odd weights, arXiv:1508.01610(2015) 
[10] A. Krieg, The Maaß-space on the Hermitian half-space of degree 2, Math. Ann., 289, 663-681(1991)

[11] T. Munemoto and S. Nagaoka, Note on $p$-adic Hermitian Eisenstein series, Abh. Math. Semin. Univ. Hambg., 76, 247-260(2006)

[12] J.D. Martin and J. Senadheera, Differential operators for Hermitian Jacobi forms and Hermitian modular forms, The Ramanujan J. 42, 443-451(2017)

[13] S. Nagaoka and S. Takemori, Notes on theta series for Niemeier lattices, The Ramanujan J. 42, 385-400(2017)

[14] S. Nagaoka and S. Takemori, On the mod p kernel of the theta operator and Eisenstein series, J. Number Theory, 188, 281-298(2018)

Shoyu Nagaoka

Dept. Mathematics Kindai Univ.

Higashi-Osaka, Osaka 577-8502, Japan

Sho Takemori

Max Planck Institut für Mathematik,

Vivatsgasse 7, 53111 Bonn, Germany 